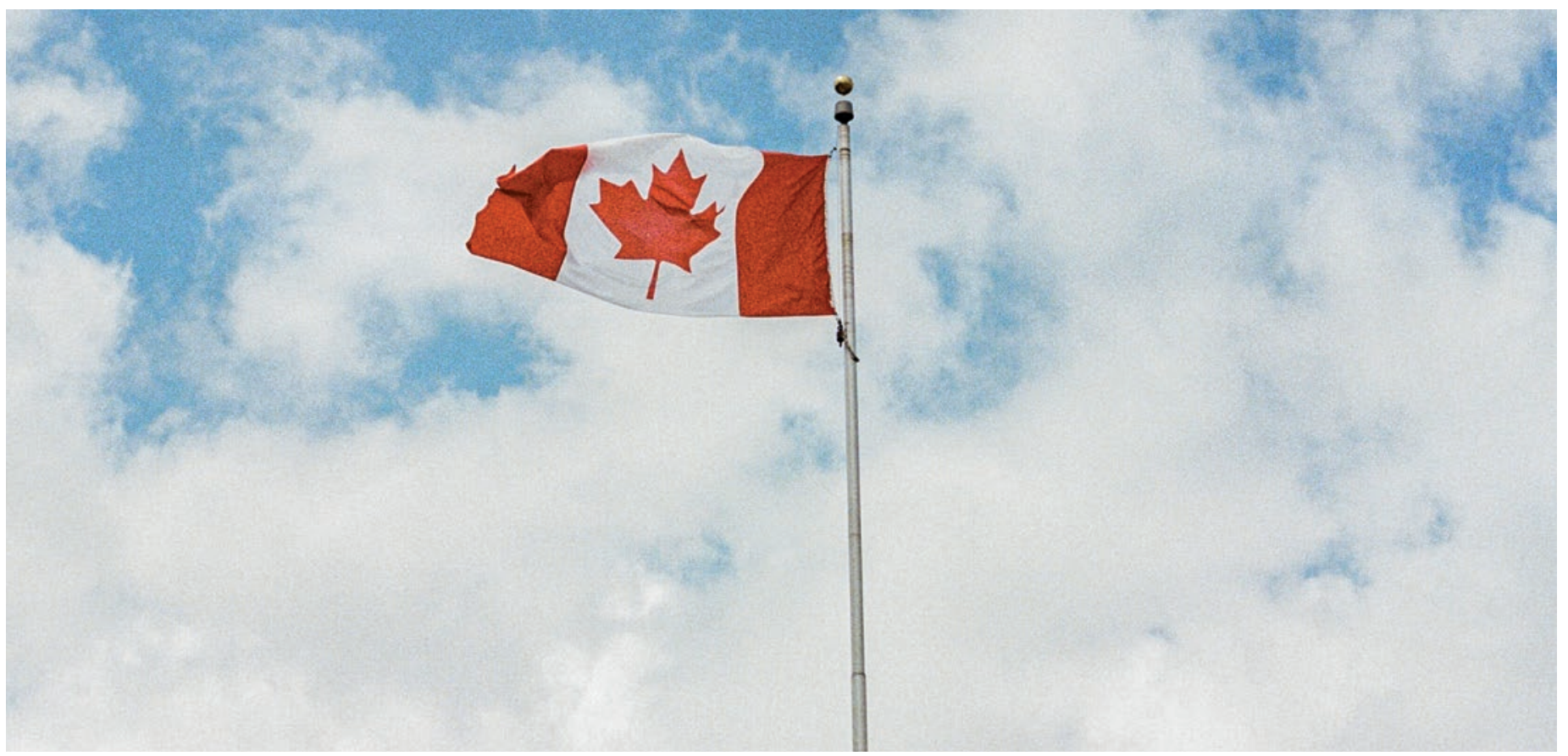

\title{
Formation interprofessionnelle et pratique collaborative au Canada
}

\section{Marie-Andrée Girarda, John H. V. Gilbert ${ }^{b}$}

a Doctorante en droit, Université de Montréal, Professeure adjointe de clinique, Faculté de Médecine, Université de Montréal; ${ }^{b}$ Professor Emeritus, University of British Columbia. Senior Scholar, WHO Collaborating Centre on Health Workforce Planning \& Research, Dalhousie University. Adjunct Professor, University of Technology, Sydney. Founding Chair, Canadian Interprofessional Health Collaborative.

Au Canada, l'interprofessionnalité dans la santé est une réalité vécue au quotidien depuis une trentaine d'année déjà, alors qu'elle peine à faire sa place en Suisse. Comment le modèle canadien fonctionne-t-il? Sur quoi son succès repose-t-il? Aperçu d'un système éprouvé, mais complexe car morcelé entre provinces et deux langues officielles, des particularités qui rappellent celles de la Suisse.

Au Canada, les systèmes de soins de santé et de services sociaux sont du ressort des provinces, bien qu'ils soient en partie financés par le Gouvernement fédéral par le biais de la législation contenue dans la Loi canadienne sur la santé [1]. La législation encadrant les professionnels de la santé et les soins étant basée au niveau provincial, le "système canadien" de santé est dans les faits constitué de treize systèmes différents et autonomes. Malgré cette autonomie, une certaine standardisation au niveau fédéral existe en raison des critères de financement qui exigent entre autres l'accès gratuit (public) aux soins nécessaires du point de vue médical et aux établissements de soins aigus, indépen- damment de la maladie ou de la capacité de paiement, et en raison de l'obligation de mobilité interprovinciale de la main d'œuvre dans l'Accord de libre-échange canadien [2]. Nonobstant cette harmonisation, les systèmes de santé et de services sociaux restent cloisonnés entre les provinces.

Ajouté à cela, le Canada a deux langues officielles, ce qui vient complexifier les relations fédérales-provinciales. Une province étant officiellement francophone, une autre officiellement bilingue, huit provinces et trois territoires officiellement anglophones, les initiatives d'harmonisation pancanadiennes nécessitent un processus constant de traduction dans les deux lan- 
gues officielles. Ces problèmes linguistiques interprovinciaux accentuent les obstacles liés au recueil et à l'interprétation des données de santé dans le vaste contexte canadien, ne donnant qu'une vision partielle. Finalement, au Canada, les organismes provinciaux sont responsables de l'éducation, y compris l'enseignement supérieur, et de la régulation des professions. Ainsi, l'accès à l'éducation professionnelle en santé et en services sociaux, la disponibilité de tels programmes et la régulation des professions de la santé et des services sociaux varient d'une province à l'autre et d'un territoire à l'autre. Cette tension constante entre instances gouvernementales fédérales et provinciales requiert un certain degré de collaboration en vue de maximiser l'accès aux soins de santé pour les Canadiens et Canadiennes. Cette collaboration fédérale/ provinciale a constitué un défi pour faire avancer l'éducation interprofessionnelle (EIP ou IPE) au Canada, les changements dans les systèmes d'éducation et de santé/ soins sociaux étant freinés par des questions réglementaires et législatives au niveau des gouvernements provinciaux et fédéral. Malgré ces difficultés, le

Maria Driedger (31 ans), étudiante sage-femme à Hamilton, Ontario, Canada

\section{"Les autres professions doivent} mieux comprendre les compétences que nous, sages-femmes, avons»

"Chez nous, au Canada, chaque femme doit décider au début de sa grossesse si elle souhaite une sage-femme ou un médecin pour les soins prénataux et l'accouchement. II n'est pas possible de combiner les deux. Si elle choisit la sagefemme, celle-ci effectuera tous les examens pendant la grossesse. Même dans le cas d'accouchement à l'hôpital, seules les sages-femmes sont présentes. Tout cela, bien sûr, à

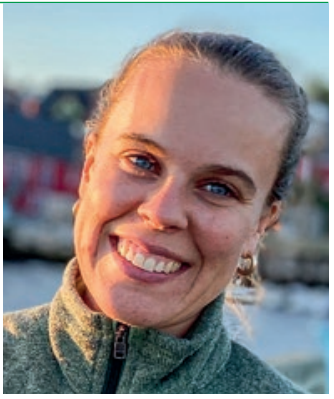

condition que la grossesse et l'accouchement se déroulent sans complications. Mais si la femme enceinte choisit un médecin, aucune sage-femme ne sera présente lors de l'accouchement et elle ne bénéficiera pas du suivi postnatal d'une sage-femme.

Cette séparation est liée au fait que la profession de sage-femme a disparu au Canada dans la première moitié du $X X^{e}$ siècle, de plus en plus de femmes accouchant à l'hôpital plutôt qu'à domicile. Ce n'est que dans le sillage du mouvement féministe, à partir des années 1970, que des militantes se sont engagées pour le retour des sages-femmes.

Je fais actuellement un 'Bachelor of Health Science in Midwifery': c'est ainsi que s'appellent les études de sage-femme qui s'étalent sur quatre ans dans mon Université à Hamilton. Actuellement, je suis en troisième année d'études durant laquelle je fais des stages interprofessionnels: j'accompagne par exemple des infirmières, des conseillères en allaitement et je vais bientôt découvrir le travail d'une ou d'un gynécologue. Ces stages pratiques sont importants pour moi. Mais ils font également partie des études, afin que les autres professions comprennent mieux les compétences que nous, sages-femmes, possédons.

Pendant la grossesse, la plupart des femmes ne s'adressent pas à une sage-femme par le biais de leur cabinet médical, mais sur recommandation d'autres femmes. Les médecins ne voient pas souvent l'intérêt de nous déléguer des cas simples à nous sages-femmes, car ils ne gagneraient rien lors des naissances. II n'y a pas suffisamment de sages-femmes au Canada pour toutes les femmes enceintes, mais une fois qu'un médecin recommande à une femme une maison de naissance dirigée par des sages-femmes, ces dernières font tout pour l'accueillir. Une telle recommandation est un grand pas en avant pour la collaboration interprofessionnelle et un signal en faveur de notre reconnaissance. statut de l'EIP/IPE au Canada a beaucoup progressé depuis les années 1960 [3]: ces expériences peuvent aider les nombreux pays qui sont face aux mêmes défis, comme la Suisse.

\section{Quel statut au Canada?}

Depuis 1969, plusieurs organisations à travers le Canada ont introduit une certaine forme de pratique collaborative interprofessionnelle centrée sur le patient (IPECP) [3, 4]. Toutefois, la mise en pratique de l'EIP/IPE et la décision quant aux meilleures pratiques et au contenu d'un cursus réussi ont été irrégulières. Cela a été principalement chapeauté par le secteur universitaire en raison des critères d'agrément spécifiques à l'EIP/IPE pour des programmes professionnels en particulier [5-7]. A l'heure actuelle, le développement de l'IPECP ne jouit pas d'un soutien cohérent des politiques professionnelles ou administratives [8,9], bien qu'elle soit reconnue comme la pierre angulaire d'un système public de santé résilient depuis le début des années 2000 [10].

Ce soutien disparate ne signifie pas l'absence de toute aide: lentement, le système soutient de plus en plus l'IPECP. A la suite de l'introduction de critères d'agrément pour l'obtention de la certification, de nombreux organismes de régulation des professions et leurs tribunaux disciplinaires ont intégré les notions de pratique collaborative dans leur code de déontologie et ont commencé à sanctionner les comportements entravant les soins en équipe [11-13]. Certains gouvernements provinciaux ont modifié le cadre législatif de leurs corps de métier afin de le rendre plus favorable à la pratique collaborative et de casser les barrières entre les professions $[12,14,15]$. Les nouvelles réglementations ont permis de transférer et de partager des tâches, ce qui donne du crédit à la notion de compétence partagée et de recoupement du champ d'activités $[12,13]$.

\section{Une pratique qui prend de l'ampleur}

Le Consortium pancanadien pour l'interprofessionnalisme en santé (CIHC-CPIS) a mis sur pied un réseau de chercheurs et praticiens intéressés par l'EIP/IPE au Canada. Pour connaître l'état actuel de celle-ci, le CPIS a mené en 2020 une analyse situationnelle d'un certain nombre de programmes universitaires à travers le pays.

Face à la diversité des parcours de formation et des établissements d'enseignement, il n'est pas surprenant que les structures organisationnelles et les programmes d'études le soient également: ainsi, certains éta- 
blissements ont intégré la formation interprofessionnelle au programme d'études d'une seule profession sans ressources spécifiquement allouées, d'autres disposent d'un bureau spécialement dédié (Rapport d'analyse de la situation du CPIS, données non publiées). Une enquête datant de 2010 avait déjà mis en évidence un processus d'appropriation institutionnelle lors de la mise en œuvre des programmes liés à l'EIP/IPE, processus respectant l'environnement particulier de chaque établissement [3].

Des points de convergence existent néanmoins. Une majorité des répondants à l'analyse de 2020 a fait état de l'existence d'un programme d'EIP/IPE (webinaires, apprentissage virtuel): on peut donc affirmer que le nombre de professionnels de la santé formés à l'IPECP est en augmentation. D'après les réponses à l'enquête, cette pratique semble faire désormais partie du programme d'études général des professionnels de la santé au Canada. Cela peut s'expliquer par l'introduction de normes d'agrément liées à la pratique collaborative dans de nombreuses professions $[7,16]$.

La majorité des répondants rapportant l'existence de tels programmes ont indiqué que leurs facilitateurs et étudiants sont issus des soins infirmiers, de la méde-

Hermann Dueck (65 ans), aumônier de formation, travaille dans un home à Winnipeg, Manitoba, au Canada, et assure régulièrement des services de garde à I'hôpital.

"Nous les aumôniers contribuons
à la santé globale»
"Au Canada, la santé est définie comme le bien-être du
corps, de l'âme et de l'esprit. Les aumôniers d'hôpital formés
comme moi s'occupent de l'état émotionnel et spirituel des
patientes et des patients, participent également aux discus-
sions de cas interprofessionnelles en qualité d'experts dans
ces domaines. Le personnel soignant est encouragé à recom-
mander notre service, mais il s'agit d'une offre volontaire.
Après avoir discuté avec une patiente ou un patient, nous
Après avoir discuté avec une patiente ou un patient, nous

laissons des commentaires dans le dossier médical afin que les médecins et le personnel soignant sachent comment s'est déroulé l'entretien et dans quel état émotionnel se trouve la personne. Supposons que je parle à un couple dont l'enfant est décédé peu après la naissance. Je décris ensuite la conversation de la manière la plus objective possible sans enfreindre le secret professionnel. Par exemple: 'Le couple était profondément bouleversé, je les ai encouragés à me raconter leur expérience et ce qu'ils ressentaient. J'ai proposé de les écouter avec empathie, j'ai passé 30 minutes avec eux et ma présence a été très appréciée. Un autre entretien est prévu.'

De telles informations sont importantes pour que le personnel soignant puisse tenir compte de l'état général des patientes et des patients. La plupart des médecins apprécient beaucoup notre travail, mais bien entendu, il $y$ en a toujours qui nous tolèrent tout au plus. Deux exemples: une fois, un médecin d'une unité de soins palliatifs a attendu longtemps devant la chambre du patient jusqu'à ce que je sorte. II ne voulait pas nous déranger parce qu'il considérait ma tâche comme plus importante que la sienne à ce moment-là. Dans une unité de soins intensifs, un médecin m'a dit un jour qu'il suffisait de donner les bons médicaments au patient qui avait fait une overdose et que tout irait bien. J'ai prudemment objecté qu'une véritable guérison ne passait pas toujours que par les médicaments. En tant qu'êtres humains, nous ne sommes pas un simple conglomérat d'organes qui doivent bien fonctionner pour que nous nous sentions bien. C'est pourquoi, nous, les aumôniers, contribuons à la santé globale.» cine, de l'ergothérapie, de la physiothérapie, bien que plus 25 professions soient représentées dans les programmes d'EIP/IPE (Rapport d'analyse de la situation du CIHC, données non publiées). Depuis le projet de l'Accreditation of interprofessional health education (AIPHE) au début des années 2000, de plus en plus de professions soumises à l'accréditation nationale sont désormais dotées de critères d'accréditation en lien avec l'EIP/IPE. Il est donc très intéressant que ces programmes implantent les notions d'IPECP dans les programmes éducatifs eux-mêmes: cela montre une valorisation et une intégration réussie de la formation interprofessionnelle [7].

Sur le plan politique, les organismes de réglementation professionnelle et les Ministères de la santé s'engagent de plus en plus dans le développement de l'IPECP. Ces décideurs politiques intègrent peu à peu les concepts de formation et de pratique collaborative à leurs directives internes, à leur code d'éthique ou prennent des mesures pour promouvoir la formation interprofessionnelle et réduire les obstacles juridiques à celle-ci $[8,17-19]$.

\section{Deux objectifs majeurs à atteindre}

Dans son plan stratégique pour 2024 établi en 2018, le CPIS veut promouvoir un réseau solide de partenaires, de leaders et de collaborateurs, dans et hors des disciplines de la santé et du domaine social, afin de fournir des données probantes et des ressources novatrices qui puissent changer la formation, le leadership, la pratique, les politiques, la réglementation et la législation. Pour assurer l'avenir, le CPIS estime qu'il est urgent d'intégrer la voix des praticiens et de s'engager avec le Patient Voices Network. De multiples projets en lien avec ces objectifs sont en cours: a) mise à jour du cadre de compétences du CPIS, b) soutien de tous les intervenants afin de s'assurer que l'agrément des programmes, tant académiques que cliniques, reconnaissent le rôle central de l'IPECP, c) développement d'un modèle d'adhésion afin d'assurer une base financière solide et d) soutien au rôle primordial que jouent les étudiants en tant que défenseurs de la formation interprofessionnelle.

Actuellement, le CPIS est le seul porte-parole des connaissances, de la formation et de la pratique interprofessionnelles au Canada. Sa mission est de développer, de partager et de défendre les pratiques les plus prometteuses pour la valeur et l'efficacité de la collaboration interprofessionnelle dans la politique, la pratique, la réglementation et l'éducation en matière de santé. Pour réaliser sa mission, le CPIS s'est engagé à continuer à: 


\section{Références} Liste complète des références sous www. bullmed.ch ou via code QR

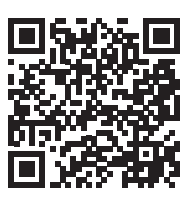

John H. V. Gilbert johnhvg[at]mail.ubc.ca

Marie-Andrée Girard marie-andree.girard.1[at] umontreal.ca
1. Promouvoir la viabilité des programmes de formation interprofessionnelle dans les universités et les instituts de tout le Canada:

a. en assurant un soutien financier répété, la promotion de postes permanents et une recherche continue,

b. en intégrant la capacité de collaboration dans le leadership des soins de santé et des services sociaux,

c. en assurant la pertinence des critères d'accréditation de la formation interprofessionnelle en termes de changement culturel dans le cursus des professionnels de la santé et des services sociaux;

2. Promouvoir et renforcer la politique en faveur de l'IPECP et minimiser les obstacles juridiques et politiques à celle-ci;

3. Promouvoir la formation interprofessionnelle et la pratique collaborative comme outils permettant de résoudre l'accès aux soins dans les zones rurales et la pénurie de professionnels de la santé et des services sociaux dans ces régions;

4. Promouvoir l'IPECP en tant qu'élément-clé de la force et de la résilience de la main d'œuvre;

5. Mener des analyses sur l'état et le statut de la FPI au Canada et sur les preuves de bonnes pratiques.

\section{Modèle canadien reconnu loin à la ronde}

Bien que les concepts de l'IPECP aient été élaborés à l'Université de la Colombie-Britannique au Canada en 1964, c'est le rapport de la Commission royale sur l'avenir des soins de santé au Canada publié fin 2002, qui recommandait des changements radicaux pour assurer la pérennité du système de soins de santé du Canada, qui a conduit à la reconnaissance de l'IPECP comme principe fondamental des systèmes de soins de santé et de services sociaux canadiens. Grâce à ce rapport, Santé Canada a financé pendant quatre ans des projets d'EIP/IPE dans tout le pays, ce qui a permis de mettre en place un certain nombre de programmes académiques et cliniques qui ont constitué la base d'un système toujours en place, de réaliser les travaux sur le cadre de compétences du CPIS et les travaux sur l'agrément (AIPHE), reconnus à l'international. L'élaboration de politiques et de procédures a également donné lieu à de nombreuses publications qui ont influencé l'IPECP à l'échelle mondiale. Par le biais d'Interprofessional. Global, le CPIS a créé des liens avec des membres et des partenaires partout dans le monde et a été une force de cohésion décisive pour le soutien à l'intégration de la collaboration interprofessionnelle dans les cadres politiques, réglementaires, législatifs, de pratique et de formation. En soutenant la série de conférence «Collaborating across Borders" avec son homologue américain (AIHC), le CPIS a aussi soutenu la dissémination de données probantes.

Dans le cadre du développement de la formation interprofessionnelle dans le continuum de l'éducation et des soins, le Canada et la Suisse sont confrontés à des défis similaires. Dans ce contexte, les expériences du Canada pourraient être utiles aux initiatives en cours en Suisse.

Crédits photo

Jp Valery / Unsplash

\section{Série sur l'interprofessionnalité}

La collaboration entre les spécialistes de différentes professions de la santé est considérée comme un levier important afin de relever les défis du système de santé. Où en est la Suisse dans ce domaine? Quels avantages apporte l'interprofessionnalité et quelles en sont les limites? Nous éclairons le sujet sous divers angles dans une série d'articles. 


\section{Références}

1 Loi Canadienne Sur La Santé.

2 LC 2017, c 33, art 219 | Loi de mise en œuvre de l'Accord de libre-échange canadien. Accessed November 30, 2021. https://www.canlii.org/fr/ca/ legis/lois/lc-2017-c-33-art-219/derniere/lc-2017-c-33-art-219.html?resultIndex=12

3 Gilbert J.H.V. The status of interprofessional education in Canada. J Allied Health. 2010;39(SUPPL. 1):216-23.

4 McCreary JF. The Education of Physicians in Canada. Can Med Assoc J. 1964;90(21):1215-21.

5 Accreditation of Interprofessional Health Education (AIPHE). Accreditation of interprofessional health education (AIPHE) principles and practices for integrating interprofessional education into the accreditation standards for six health professions in Canada. Retrieved from. Published online 2009. http://www.cihc.ca/aiphe/

6 Accreditation of Interprofessional Health Education (AIPHE). Interprofessional Health Education Accreditation Standards Guide Phase 2. Published online 2012. http://www.cihc.ca/files/resources/public/English/AIPHE\%2OInterprofessional\%2OHealth\%2OEducation\%20 Accreditation\%20Standards\%20Guide_EN.pdf

7 Azzam M, Puvirajah A, Girard MA, Grymonpre RE. Interprofessional education-relevant accreditation standards in Canada: a comparative document analysis. Human Resources for Health. 2021;19(1):66. doi:10.1186/s12960-021-00611-1

8 Girard MA, Régis C, Denis JL. Interprofessional collaboration and health policy: results from a Quebec mixed method legal research. Journal of Interprofessional Care. Published online May 6, 2021:1-8. doi:10.1080/13561820.2021.1891030

9 Suter E, Mallinson S, Misfeldt R, Boakye O, Nasmith L, Wong ST. Advancing team-based primary health care: a comparative analysis of policies in western Canada. BMC Health Services Research. 2017;17(1). doi:10.1186/s12913-017-2439-1

10 Romanow RJ, Commission sur l'avenir des soins de santé au Canada. Guidé par nos valeurs l'avenir des soins de santé au Canada: rapport final. Commission sur l'avenir des soins de santé au Canada; 2002. Accessed May 16, 2016. http://dsp-psd.communication.gc.ca/Collection/ CP32-85-2002F.pdf

11 Trudeau JB, de Grandmont S, Lafrance L, Poitras L. la "loi 90»: la force de l’interdisciplinarité. Des connaissances et des compétences à partager. In: Le Système Socio-Sanitaire Au Québec; Gouvernance, Régulation et Participation. Gaëtan Morin Editeur; 2007:263-72.

12 Bélanger L, Roy C, Simard MC, Trudeau JB. Partager des activités professionnelles pour mieux collaborer. Service de la formation continue du Barreau du Québec, 2019. 2019; Développements récents en droit de la santé (2019) (EYB2019DEV2790).

13 Bourgeault IL, Grignon M. A comparison of the regulation of health professional boundaries across OECD countries. The European Journal of Comparative Economics. 2013;10(2):199-224.

14 Lahey W, Fierlbeck K. Legislating collaborative self-regulation in Canada: A comparative policy analysis. Journal of Interprofessional Care. 2016;30(2):211-6. doi:10.3109/13561820.2015.1109501

15 Lahey W. Legislating Interprofessional Regulatory Collaboration in Nova Scotia. Health Reform Observer-Observatoire des Réformes de Santé. 2013;1(1). doi:10.13162/hro-ors.01.01.04

16 Curran V. Environmental scan report: Interprofessional education and accreditation processes in pre-licensure health professional education Canadian Interprofessional Health Collaborative URL: http://www cihc ca/files/aiphe/resources/AIPHE\% 20Environmental\% 20Scan\% 20Report pdf. Published online 2008. Accessed March 21, 2016. http://www.cihc.ca/files/resources/public/English/AIPHE\%20Environmental\%20Scan\%20 Report.pdf

17 Girard MA. Interprofessional education and collaborative practice policies and law: an international review and reflective questions. Human Resources for Health. 2021;19(1):9. doi:10.1186/s12960-020-00549-w

18 Girard MA. Interprofessional Collaborative Practice and Law: A Reflective Analysis of 14 Regulation Structures. Journal of Research in Interprofessional Practice and Education. 2019;9(2).

19 Ries NM. Law matters: How the legal context in Canada influences interprofessional collaboration. Journal of Interprofessional Care. 2017;31(4):417-419. doi:10.1080/13561820.2017.1310495 